Pacific

Journal of

Mathematics

ATTRACTORS FOR STRONGLY DAMPED WAVE EQUATIONS WITH CRITICAL NONLINEARITIES

Alexandre N. Carvalho and Jan W. Cholewa 


\title{
ATTRACTORS FOR STRONGLY DAMPED WAVE EQUATIONS WITH CRITICAL NONLINEARITIES
}

\author{
Alexandre N. Carvalho and Jan W. Cholewa
}

In this paper we obtain global well-posedness results for the strongly damped wave equation $u_{t t}+(-\Delta)^{\theta} u_{t}=\Delta u+f(u)$, for $\theta \in\left[\frac{1}{2}, 1\right]$, in $H_{0}^{1}(\Omega) \times L^{2}(\Omega)$ when $\Omega$ is a bounded smooth domain and the map $f$ grows like $|u|^{\frac{n+2}{n-2}}$. If $f=0$, then this equation generates an analytic semigroup with generator $-\mathcal{A}_{(\theta)}$. Special attention is devoted to the case when $\theta=1$ since in this case the generator $-\mathcal{A}_{(1)}$ does not have compact resolvent, contrary to the case $\theta \in\left[\frac{1}{2}, 1\right)$. Under the dissipativeness condition $\lim \sup _{|s| \rightarrow \infty} \frac{f(s)}{s} \leq 0$ we prove the existence of compact global attractors for this problem. In the critical growth case we use Alekseev's nonlinear variation of constants formula to obtain that the semigroup is asymptotically smooth.

\section{Introduction.}

For $\theta \in\left[\frac{1}{2}, 1\right], \eta>0$, we consider the global well-posedness and existence of global attractors for a family of problems of the form

$$
\left\{\begin{array}{l}
u_{t t}+\eta(-\Delta)^{\theta} u_{t}+(-\Delta) u=f(u), t>0, x \in \Omega, \\
u(0, x)=u_{0}(x), u_{t}(0, x)=v_{0}(x), x \in \Omega, \\
u(t, x)=0, t \geq 0, x \in \partial \Omega,
\end{array}\right.
$$

where $\Omega$ is a bounded $C^{2}$ smooth domain in $\mathbb{R}^{n}$ and $n \geq 3$. We write further $A$ for $-\Delta$ with the Dirichlet boundary conditions. It is well-known that $A$ is a positive, self-adjoint operator with the domain $D(A)=H^{2}(\Omega) \cap H_{0}^{1}(\Omega)$ and $-A$ generates an analytic semigroup on $X=X^{0}=L^{2}(\Omega)$. We denote by $X^{\alpha}$ the fractional power spaces associated to the operator $A$; that is $X^{\alpha}=D\left(A^{\alpha}\right)$ endowed with the graph norm.

The problems (1) will be viewed as ordinary differential equations in a product space $Y=Y^{0}=X^{\frac{1}{2}} \times X^{0}$ :

$$
\frac{d}{d t}\left[\begin{array}{l}
u \\
v
\end{array}\right]+\mathcal{A}_{(\theta)}\left[\begin{array}{l}
u \\
v
\end{array}\right]=\mathcal{F}\left(\left[\begin{array}{l}
u \\
v
\end{array}\right]\right), t>0,\left[\begin{array}{l}
u \\
v
\end{array}\right]_{t=0}=\left[\begin{array}{l}
u_{0} \\
v_{0}
\end{array}\right] .
$$


Here $\mathcal{A}_{(\theta)}: D\left(\mathcal{A}_{(\theta)}\right) \subset Y^{0} \rightarrow Y^{0}$ and $\mathcal{F}$ are given by

$$
\begin{array}{r}
\mathcal{A}_{(\theta)}\left[\begin{array}{l}
\varphi \\
\psi
\end{array}\right]=\left[\begin{array}{c}
-\psi \\
A^{\theta}\left(A^{1-\theta} \varphi+\eta \psi\right)
\end{array}\right] \\
\text { for }\left[\begin{array}{l}
\varphi \\
\psi
\end{array}\right] \in D\left(\mathcal{A}_{(\theta)}\right), \quad \mathcal{F}\left(\left[\begin{array}{l}
u \\
v
\end{array}\right]\right)=\left[\begin{array}{c}
0 \\
F(u)
\end{array}\right],
\end{array}
$$

where

$$
D\left(\mathcal{A}_{(\theta)}\right)=Y_{(\theta)}^{1}=\left\{\left[\begin{array}{l}
\varphi \\
\psi
\end{array}\right] \in X^{\frac{3}{2}-\theta} \times X^{\frac{1}{2}}: A^{1-\theta} \varphi+\eta \psi \in X^{\theta}\right\}, \theta \in\left[\frac{1}{2}, 1\right]
$$

and $F$ is the Nemitskiu map associated to $f(u)$. Of course,

$$
\mathcal{A}_{(\theta)}\left[\begin{array}{c}
\varphi \\
\psi
\end{array}\right]=\left[\begin{array}{c}
-\psi \\
A \varphi+\eta A^{\theta} \psi
\end{array}\right] \text { for }\left[\begin{array}{c}
\varphi \\
\psi
\end{array}\right] \in X^{1} \times X^{\theta}
$$

$X^{1} \times X^{\theta}$ being a dense subset of $D\left(\mathcal{A}_{(\theta)}\right)$.

The linear problem associated to $(2)$ in $Y^{0}$,

$$
\ddot{u}+\eta A^{\theta} \dot{u}+A u=0, t>0, u(0)=u_{0}, \dot{u}(0)=v_{0},
$$

is studied in $[\mathbf{7}, \mathbf{8}, \mathbf{9}]$, where the sectoriality of $\mathcal{A}_{(\theta)}$ is established and a description of the fractional power spaces $Y_{(\theta)}^{\alpha}, \alpha \in[0,1]$ is given.

We choose as a base space for (1) the product space $Y^{0}=X^{\frac{1}{2}} \times X^{0}$. This space seems to be the best possible to study the asymptotic behavior of (1) since in it we may exhibit an energy functional to (1).

The cases $\theta=\frac{1}{2}$ and $\theta=1$ will deserve special attention. For $\theta=\frac{1}{2}$, the form of the damping term $A^{\frac{1}{2}} u_{t}$ allows us to obtain a more complete description of the fractional power spaces associated to $\mathcal{A}_{\left(\frac{1}{2}\right)}$. Using this, we are able to describe the extrapolated fractional power scale generated by $\left(Y^{0}, \mathcal{A}_{\left(\frac{1}{2}\right)}\right)$ (see [5]) and obtain the convergence of bounded sets from $Y^{0}$ to the attractor in the strong topology of $H^{1+\alpha}(\Omega) \times H^{\alpha}(\Omega)$-norm, $\alpha \in$ $\left[\frac{n-2}{n+2}, 1\right)$. For $\theta=1$ we have that the nonlinearity becomes subcritical, nevertheless, we loose compactness of the semigroup and of the nonlinearity (so that subcritical is of no help). However, in this latter case we are still able to ensure the existence of a compact global attractor with the aid of a nonlinear variation of constants formula.

The crucial result of [5] that we will use here is that:

Theorem 1. If $f$ satisfies

$$
\left|f(u)-f\left(u^{\prime}\right)\right| \leq c\left|u-u^{\prime}\right|\left(1+|u|^{\rho-1}+\left|u^{\prime}\right|^{\rho-1}\right)
$$

with $\rho \leq \frac{n+2}{n-2}$, then (1) is locally well-posed in $H_{0}^{1}(\Omega) \times L^{2}(\Omega)$. 
In the present paper our main concern is the asymptotic behavior of (1) and a great deal of our effort will go into the following conjecture.

Conjecture. If in addition to (6) $f$ satisfies the dissipativeness condition

$$
\limsup _{|u| \rightarrow \infty} \frac{f(u)}{u} \leq 0
$$

then the problem (1) with $\theta \in\left[\frac{1}{2}, 1\right]$ has a compact global attractor.

The paper is organized as follows. In Section 2 we briefly recall the results of [5] concerning solvability of (1). Section 3 is devoted to a discussion of the additional regularity of the solutions to (1). In Section 4 we prove the global solvability and the existence of global attractors for (1), i.e.,

- in Subsection 4.1 we study the existence of a compact global attractor for the case $\theta=\left[\frac{1}{2}, 1\right), \rho<\frac{n+2}{n-2}$ and $f$ satisfying (6), (7),

- in Subsection 4.2 we treat the subcritical case $\rho<\frac{n+2}{n-2}$ for $\theta=1$,

- in Subsection 4.3 we deal with the critical case $\rho=\frac{n+2}{n-2}$ for $\theta=1$.

We remark that for $\theta=1$ the resolvent of $\mathcal{A}_{(\theta)}$ is not compact. However, we are able to show that the semigroup $\{T(t)\}$ corresponding to (1) is asymptotically smooth decomposing $\{T(t)\}$ on a sum of the exponentially decaying semigroup and a family of compact maps (see [11]). In the subcritical case this may be accomplished based on compactness of the nonlinear term. In the critical case the latter argument cannot be used and to overcome this difficulty we employ the nonlinear variation of constants formula as in [3].

\section{Local solvability of (1) in $Y^{0}$.}

We start with the results of [5] on local well-posedness and regularity for (2) with initial conditions in $Y^{0}$ and nonlinearities growing critically. Recall that (see [5, Propositions 1, 4]):

Proposition 1. $\mathcal{A}_{(\theta)}, \theta \in\left[\frac{1}{2}, 1\right]$, is a sectorial, positive operator in $Y^{0}$. The semigroup of contractions $\left\{e^{-\mathcal{A}_{(\theta)} t}\right\}$ is analytic in $Y_{(\theta)}^{\alpha}, \alpha \in[0,1)$. It is also compact for $t>0$ except the case $\theta=1$. Furthermore,

$$
Y_{(\theta)}^{\alpha}=\left[Y^{0}, Y_{(\theta)}^{1}\right]_{\alpha}= \begin{cases}X^{\frac{1}{2}+\alpha(1-\theta)} \times X^{\theta \alpha}, & \alpha \in\left[0, \frac{1}{2}\right], \\
{\left[\begin{array}{c}
\varphi \\
\psi
\end{array}\right] \in X^{\frac{1}{2}+\alpha(1-\theta)} \times X^{\theta-\frac{1}{2}+\alpha(1-\theta)}:} & \\
\left.A^{1-\theta} \varphi+\eta \psi \in X^{\theta \alpha}\right\}, & \alpha \in\left[\frac{1}{2}, 1\right] .\end{cases}
$$


Following [1], we denote by $Y_{(\theta)_{-1}}, \theta \in\left[\frac{1}{2}, 1\right]$, the extrapolated space of $Y^{0}$ generated by $\mathcal{A}_{(\theta)}$ which is the completion of the normed space $\left(Y^{0}, \| \mathcal{A}_{(\theta)}^{-1}\right.$. $\left.\|_{Y^{0}}\right)$. It was shown in [5, Proposition 5] that:

- $\mathcal{A}_{(\theta)_{-1}}\left(\mathcal{A}_{(\theta)_{-1}}\right.$ being the closure of $\mathcal{A}_{(\theta)}$ in $\left.Y_{(\theta)_{-1}}\right)$ is sectorial and positive operator in $Y_{(\theta)_{-1}}$ with $D\left(\mathcal{A}_{(\theta)_{-1}}\right)=Y_{(\theta)_{-1}}^{1}=Y^{0}$,

- imaginary powers of $\mathcal{A}_{(\theta)_{-1}}$ are bounded,

- $\mathcal{A}_{(\theta)_{-1}}$ has compact resolvent except for $\theta=1$.

When dealing with fractional powers it is important to know the embeddings that relate the spaces in the fractional power scale and the known spaces. Result below comes from $[5, \S 2.2, \S 2.3]$ and will be needed to obtain regularity and asymptotic compactness of the semigroup generated by (2).

Lemma 1. Let $\left[\left(X^{\alpha}, A_{\alpha}\right), \alpha \in \mathbb{R}\right]\left(A_{\alpha}\right.$ being the realization of $A$ in $\left.X^{\alpha}\right)$ be generated by $\left(L^{2}(\Omega),\left(-\Delta_{D}\right)\right)$. Then:

(i) For $\alpha(1-\theta)<\frac{1}{4}$ and $n=3$ or $n>3$,

$$
Y_{(\theta)_{-1}}^{1+\alpha} \subset H^{1+2 \alpha(1-\theta)}(\Omega) \times H^{2 \theta \alpha}(\Omega) \subset L^{q_{1}}(\Omega) \times L^{q_{2}}(\Omega),
$$

provided that $1 \leq q_{1} \leq \frac{2 n}{n-2-4 \alpha(1-\theta)}, 1 \leq q_{2} \leq \frac{2 n}{n-4 \alpha \theta}, \alpha \in\left[0, \frac{1}{2}\right], \theta \in$ $\left[\frac{1}{2}, 1\right]$

(ii) for $n=3$ and $\alpha=\theta=\frac{1}{2}$ the embedding (9) holds for $1 \leq q_{1} \leq \infty$, $1 \leq q_{2} \leq 3$

(iii) for $n \geq 3$ we have $Y_{\left(\frac{1}{2}\right)_{-1}}^{1+\alpha} \subset H^{1+\alpha}(\Omega) \times H^{\alpha}(\Omega)$ for any $\alpha \in[0,1]$; furthermore,

$$
\begin{gathered}
\left\{\begin{array}{l}
Y_{(\theta)_{-1}}^{\alpha} \supset X^{\frac{1}{2}-(1-\alpha)(1-\theta)} \times X^{-\frac{1}{2}+\alpha(1-\theta)} \supset X^{\frac{1}{2}-(1-\alpha)(1-\theta)} \times L^{q}(\Omega), \\
\text { if } q \geq \frac{2 n}{n+2-4 \alpha(1-\theta)}, \alpha \in\left[0, \frac{1}{2}\right], \theta \in\left[\frac{1}{2}, 1\right], n \geq 3,
\end{array}\right. \\
\text { whereas } Y_{\left(\frac{1}{2}\right)_{-1}}^{\alpha}=X^{\frac{\alpha}{2}}(\Omega) \times X^{\frac{\alpha-1}{2}}(\Omega) \text { for any } \alpha \in[0,1], n \geq 3 .
\end{gathered}
$$

Following [5] we shall next study (1) as a sectorial problem (11) in $Y_{(\theta)_{-1}}$, $\theta \in\left[\frac{1}{2}, 1\right]$,

$$
\frac{d}{d t}\left[\begin{array}{l}
u \\
v
\end{array}\right]+\mathcal{A}_{(\theta)_{-1}}\left[\begin{array}{l}
u \\
v
\end{array}\right]=\mathcal{F}\left(\left[\begin{array}{l}
u \\
v
\end{array}\right]\right), t>0,\left[\begin{array}{l}
u \\
v
\end{array}\right]_{t=0}=\left[\begin{array}{l}
u_{0} \\
v_{0}
\end{array}\right] .
$$

Our concern will be the $\varepsilon$-regular solutions to (11) originating at the elements of $Y_{(\theta)_{-1}}^{1}=Y^{0}$ (see Definition 2).

We first recall that if $P$ is a sectorial, positive operator acting in a Banach space $Z=Z^{0}$ and $\varepsilon$ is a nonnegative number, then:

Definition 1. $G: D(G) \rightarrow Z$ is $\varepsilon$-regular relatively to $\left(Z^{1}, Z^{0}\right)$ (equivalently, $G$ is of class $\mathcal{F}(\varepsilon, \rho, \gamma(\varepsilon), C))$ if and only if there are constants $\rho>1, \gamma(\varepsilon) \geq$ 
$0, C>0$ such that $\rho \varepsilon \leq \gamma(\varepsilon)<1, G$ takes $Z^{1+\varepsilon}$ into $Z^{\gamma(\varepsilon)}$, and the following estimate holds:

$$
\begin{aligned}
& \left\|G\left(z_{1}\right)-G\left(z_{2}\right)\right\|_{Z^{\gamma(\varepsilon)}} \\
& \leq C\left\|z_{1}-z_{2}\right\|_{Z^{1+\varepsilon}}\left(\left\|z_{1}\right\|_{Z^{1+\varepsilon}}^{\rho-1}+\left\|z_{2}\right\|_{Z^{1+\varepsilon}}^{\rho-1}+1\right), z_{1}, z_{2} \in Z^{1+\varepsilon} .
\end{aligned}
$$

The following result of [5, Corollary 2] plays an important role in the regularity of the solutions of (1) and we will refer to it later in the paper.

Theorem 2. Assume that $f$ satisfies (6) with $1<\rho \leq \frac{n+2}{n-2}$ and let $\mathcal{F}$ be the map defined in (3). Then, $\mathcal{F}$ is an $\varepsilon$-regular map relatively to $\left(Y_{(\theta)_{-1}}^{1}, Y_{(\theta)_{-1}}\right)$ for each $\varepsilon \in\left[0, \frac{1}{2 \rho}\right]$ with $\gamma(\varepsilon)=\rho \varepsilon$, that is,

$$
\begin{array}{r}
\left\|\mathcal{F}\left(\left[\begin{array}{l}
u \\
v
\end{array}\right]\right)-\mathcal{F}\left(\left[\begin{array}{l}
u^{\prime} \\
v^{\prime}
\end{array}\right]\right)\right\|_{Y_{(\theta)_{-1}}^{\gamma(\varepsilon)}}\left(1+\left\|\left[\begin{array}{l}
u \\
v
\end{array}\right]\right\|_{Y_{(\theta)_{-1}}^{1+\varepsilon}}^{\rho-1}+\left\|\left[\begin{array}{l}
u^{\prime} \\
v^{\prime}
\end{array}\right]\right\|_{Y_{(\theta)_{-1}}^{1+\varepsilon}}^{\rho-1}\right. \\
\leq c\left\|\left[\begin{array}{l}
u \\
v
\end{array}\right]-\left[\begin{array}{l}
u^{\prime} \\
v^{\prime}
\end{array}\right]\right\| \|_{Y_{(\theta)_{-1}}^{1+\varepsilon}}\left(\begin{array}{l}
u \\
v
\end{array}\right],\left[\begin{array}{c}
u^{\prime} \\
v^{\prime}
\end{array}\right] \in Y_{(\theta)_{-1}}^{1+\varepsilon},
\end{array}
$$

for $\varepsilon \in\left[0, \frac{1}{2 \rho}\right], \gamma(\varepsilon)=\varepsilon \rho$. Moreover, if $\theta=\frac{1}{2}$, then (13) holds with $\gamma(\varepsilon)=$ $\rho \varepsilon$ for each $\varepsilon \in\left[0, \frac{1}{\rho}\right)$.

Consider now an abstract problem:

$$
\dot{z}+P z=G(z), t>0, z(0)=z_{0}
$$

( $P, G$ as above) and take $\varepsilon \geq 0, \tau>0, z_{0} \in Z^{1}$. Recall that:

Definition 2. A function $z=z\left(\cdot, z_{0}\right):[0, \tau] \rightarrow Z^{1}$ is an $\varepsilon$-regular solution to the problem (14) if and only if $z \in C\left([0, \tau], Z^{1}\right) \cap C\left((0, \tau], Z^{1+\varepsilon}\right)$, and

$$
z(t)=e^{-P t} z_{0}+\int_{0}^{t} e^{-P(t-s)} G(z(s)) d s \text { for } t \in[0, \tau] .
$$

The existence of the $\varepsilon$-regular solution to (11) under the assumptions (6) has been recently discussed in [5] based on the original results reported in $[\mathbf{2}],[\mathbf{4}]$. We thus have (see [2, Corollary 1], [5, Theorem 3]):

Theorem 3. Consider (11) as an abstract counterpart of (1) in $Y_{(\theta)_{-1}}$, $\theta \in\left[\frac{1}{2}, 1\right]$, choose $\left[\begin{array}{l}\bar{u}_{0} \\ \bar{v}_{0}\end{array}\right] \in Y^{0}$ and denote by $B_{Y^{0}}\left(\left[\begin{array}{l}\bar{u}_{0} \\ \bar{v}_{0}\end{array}\right], r\right)$ a ball in $Y^{0}$ centered at $\left[\begin{array}{l}\bar{u}_{0} \\ \bar{v}_{0}\end{array}\right]$ with radius $r>0$. Suppose further that $\mathcal{F}$ is an $\varepsilon$-regular 
map relatively to $\left(Y_{(\theta)_{-1}}^{1}, Y_{(\theta)_{-1}}\right)$ and $\varepsilon>0$. Then, there are $r>0$ and $\tau_{0}>0$ such that for each $\left[\begin{array}{l}u_{0} \\ v_{0}\end{array}\right] \in B_{Y^{0}}\left(\left[\begin{array}{l}\bar{u}_{0} \\ \bar{v}_{0}\end{array}\right], r\right)$ there exists a unique $\varepsilon$ regular solution $\left[\begin{array}{l}u \\ v\end{array}\right]\left(\cdot, u_{0}, v_{0}\right)$ to $(11)$. In addition,

(i) $t^{\zeta}\left\|\left[\begin{array}{l}u \\ v\end{array}\right]\left(t, u_{0}, v_{0}\right)\right\|_{Y_{(\theta)-1}^{1+\zeta}} \rightarrow 0$ as $t \rightarrow 0^{+}, 0<\zeta<\gamma(\varepsilon)$,

(ii) $t^{\zeta}\left\|\left[\begin{array}{l}u \\ v\end{array}\right]\left(t, u_{1}, v_{1}\right)-\left[\begin{array}{l}u \\ v\end{array}\right]\left(t, u_{2}, v_{2}\right)\right\|_{Y_{(\theta)-1}^{1+\zeta}} \leq C^{\prime}\left(\zeta_{0}\right)\left\|\left[\begin{array}{l}u_{1} \\ v_{1}\end{array}\right]-\left[\begin{array}{l}u_{2} \\ v_{2}\end{array}\right]\right\|_{Y^{0}}$ whenever $t \in\left[0, \tau_{0}\right], 0 \leq \zeta \leq \zeta_{0}<\gamma(\varepsilon),\left[\begin{array}{l}u_{1} \\ v_{1}\end{array}\right],\left[\begin{array}{l}u_{2} \\ v_{2}\end{array}\right] \in B_{Y^{0}}\left(\left[\begin{array}{l}\bar{u}_{0} \\ \bar{v}_{0}\end{array}\right], r\right)$, (iii) $\left[\begin{array}{l}u \\ v\end{array}\right]\left(\cdot, u_{0}, v_{0}\right) \in C\left(\left(0, \tau_{0}\right], Y_{(\theta)_{-1}}^{1+\gamma(\varepsilon)}\right) \cap C^{1}\left(\left(0, \tau_{0}\right], Y_{(\theta)_{-1}}^{1+\zeta}\right)$ for $0 \leq \zeta<$ $\gamma(\varepsilon)$; in particular, $\left[\begin{array}{l}u \\ v\end{array}\right]\left(\cdot, u_{0}, v_{0}\right)$ satisfies both relations in $(2)$.

Remark 1. Based on Theorem 2 one may substitute in the above Conditions (i)-(iii) numbers $\varepsilon, \gamma(\varepsilon)$ such that

$$
\begin{cases}\gamma(\varepsilon)=\frac{1}{2} & \text { if } \theta \in\left(\frac{1}{2}, 1\right], \\ \gamma(\varepsilon)<1 \text { and } \gamma(\varepsilon) \text { arbitrarily close to } 1 & \text { if } \theta=\frac{1}{2} .\end{cases}
$$

We also point out that crucial in this discussion Condition (13) holds whenever $\rho, \varepsilon, \gamma(\varepsilon)$ fulfill the restrictions

$$
1<\rho \leq \frac{n+2-4 \gamma(\varepsilon)(1-\theta)}{n-2-4 \varepsilon(1-\theta)}, 0 \leq \varepsilon \leq \frac{1}{2 \rho}, \rho \varepsilon \leq \gamma(\varepsilon) \leq \frac{1}{2},
$$

(see [5, Lemma 3] for detailed calculations). For the case $\rho<\frac{n+2}{n-2}$ this allows us to require of the numbers $\varepsilon$ and $\gamma(\varepsilon)$ in (15) to satisfy additionally the inequality $\gamma(\varepsilon)>\varepsilon \rho$; for example we may then choose

$$
\begin{cases}\varepsilon>\left(\frac{n-2}{n+2}\right)^{2}, \gamma(\varepsilon)=\varepsilon \frac{n+2}{n-2} \text { and } \varepsilon \text { sufficiently close to }\left(\frac{n-2}{n+2}\right)^{2}, & \text { if } \theta=\frac{1}{2}, \\ \max \left\{0, \frac{\rho(n-2)-(n+2)+2(1-\theta)}{4(1-\theta) \rho}\right\}<\varepsilon<\frac{1}{2 \rho}, \gamma(\varepsilon)=\frac{1}{2}, & \text { if } \frac{1}{2}<\theta<1, \\ \gamma(\varepsilon)=\frac{1}{2}, 0<\varepsilon<\frac{1}{2 \rho}, & \text { if } \theta=1 .\end{cases}
$$

Consequently, in a subcritical case a number $r$ in Theorem 3 can be chosen arbitrarily large so that the time of existence can be chosen uniform on bounded subsets of $Y_{(\theta)_{-1}}^{1}$ (see [2, Corollary 1]). 


\section{Smoothing action of $\varepsilon$-regular solutions.}

Our aim here is to show that the $\varepsilon$-regular solutions resulting from Theorem 3 are in fact smoother solutions. Namely, they may be viewed as the solutions to (2) within the approach of [12]. We shall assume that

(17) $f$ satisfies (6) with $\rho \leq \frac{n+2}{n-2}, \theta \in\left[\frac{1}{2}, \frac{n+6}{2 n+4}\right)$ and

$$
\alpha \in\left[\frac{n-2}{2(1-\theta)(n+2)}, 1\right) .
$$

Lemma 2. Suppose that (17) holds. Then the map $\mathcal{F}$ defined in (3) takes $Y_{(\theta)}^{\alpha}$ into $Y^{0}$ and is Lipschitz continuous on bounded subsets of $Y_{(\theta)}^{\alpha}$.

Proof. The proof follows by standard calculations based on the Hölder inequality and Sobolev embedding (see the description of $Y_{(\theta)}^{\alpha}$ spaces given in (8)).

The above lemma and the general results of [12] imply then solvability of (2) and consequently smoothness of the $\varepsilon$-regular solutions stated in Theorem 4 below.

Lemma 3. Under the assumptions of Lemma 2 for each $\left[\begin{array}{l}u_{0} \\ v_{0}\end{array}\right] \in Y_{(\theta)}^{\alpha}$ there exists a unique $Y_{(\theta)}^{\alpha}$-solution to (2) defined on a maximal interval of existence $\left[0, \tau_{u_{0}, v_{0}}\right)$. That is, there exists a unique function $\left[\begin{array}{l}u \\ v\end{array}\right]\left(\cdot, u_{0}, v_{0}\right) \in$ $C\left(\left[0, \tau_{u_{0}, v_{0}}\right), Y_{(\theta)}^{\alpha}\right)$ such that:
(i) $\left[\begin{array}{l}u \\ v\end{array}\right]\left(t, u_{0}, v_{0}\right) \in C\left(\left(0, \tau_{u_{0}, v_{0}}\right), Y_{(\theta)}^{1}\right)$,
(ii) $\left[\begin{array}{l}u \\ v\end{array}\right]\left(\cdot, u_{0}, v_{0}\right) \in C^{1}\left(\left(0, \tau_{u_{0}, v_{0}}\right), Y_{(\theta)}^{\beta}\right), \beta \in[0,1)$,
(iii) both relations in (2) are satisfied.

Theorem 4. If, in addition to (17), we assume that

$$
\text { either } n \geq 3 \text { and } \theta=\frac{1}{2} \text { or } 3 \leq n \leq 5 \text { and } \theta \in\left(\frac{1}{2}, \frac{4}{n+2}\right) \text {, }
$$

then the $\varepsilon$-regular solutions from Theorem 3 fulfill Conditions (i)-(iii) of Lemma 3.

Proof. Choose $\varepsilon>0$ in Theorem 2 such that

$$
\begin{cases}\gamma(\varepsilon) \in\left(\frac{n-2}{n+2}, 1\right) & \text { if } \theta=\frac{1}{2}, n \geq 3, \\ \gamma(\varepsilon)=\frac{1}{2}>\frac{n-2}{2(1-\theta)(n+2)} & \text { if } \theta \in\left(\frac{1}{2}, \frac{4}{n+2}\right), 3 \leq n \leq 5,\end{cases}
$$


and let $\left[\begin{array}{l}u \\ v\end{array}\right]\left(\cdot, u_{0}, v_{0}\right)$ be $\varepsilon$-regular solution obtained in Theorem 3 (see Remark 1). Since $Y_{(\theta)_{-1}}^{1+\gamma(\varepsilon)}=Y_{(\theta)}^{\gamma(\varepsilon)} \subset Y_{(\theta)}^{\frac{n-2}{2(1-\theta)(n+2)}}$ we find from Theorem 3(iii) that

$$
\left[\begin{array}{l}
u \\
v
\end{array}\right]\left(s, u_{0}, v_{0}\right) \in Y_{(\theta)}^{\frac{n-2}{2(1-\theta)(n+2)}} \text { for each } s \in\left(0, \tau_{0}\right) \text {. }
$$

According to Lemma 3 there exists $Y_{(\theta)}^{\frac{n-2}{2(1-\theta)(n+2)}}$-solution $\left[\begin{array}{l}\widetilde{u} \\ \widetilde{v}\end{array}\right]\left(\cdot, u\left(s, u_{0}, v_{0}\right)\right.$, $\left.v\left(s, u_{0}, v_{0}\right)\right)$ to $(1)$. This proves that

$$
\left[\begin{array}{l}
u \\
v
\end{array}\right]\left(t+s, u_{0}, v_{0}\right)=\left[\begin{array}{l}
\widetilde{u} \\
\widetilde{v}
\end{array}\right]\left(t, u\left(s, u_{0}, v_{0}\right), v\left(s, u_{0}, v_{0}\right)\right), t \in\left[0, \tau_{u_{0}, v_{0}}\right),
$$

and consequently,

$$
\begin{aligned}
& {\left[\begin{array}{l}
u \\
v
\end{array}\right]\left(t, u_{0}, v_{0}\right) \in Y_{(\theta)}^{1}, t \in\left(s, \tau_{u_{0}, v_{0}}\right),} \\
& \left.\qquad \begin{array}{l}
u \\
v
\end{array}\right]\left(\cdot, u_{0}, v_{0}\right) \in C^{1}\left(\left(s, \tau_{u_{0}, v_{0}}\right), Y_{(\theta)}^{\beta}\right), \quad \beta \in[0,1) .
\end{aligned}
$$

Since $s>0$ could be arbitrarily small, the proof is complete.

\section{Global solvability of (2) and a global attractor.}

4.1. Subcritical case: $\theta \in\left[\frac{1}{2}, 1\right)$. In this subsection we consider the existence of a compact global attractor for (2) when the growth of $f$ is subcritical; that is, (6) holds with $\rho<\frac{n+2}{n-2}$. We first restrict our attention to the cases mentioned in (18) when either $\theta=\frac{1}{2}$ and $n \geq 3$ or $\theta \in\left(\frac{1}{2}, \frac{4}{n+2}\right)$ and $3 \leq n \leq 5$.

Lemma 4. If (18) holds and $f$ satisfies (6) with $\rho<\frac{n+2}{n-2}$, then for any bounded set $B \subset Y^{0}$ there are a time $\tau_{B}>0$ and the numbers $\varepsilon>0$, $\gamma(\varepsilon)>\rho \varepsilon$ as in (16) such that the $\varepsilon$-regular solutions $\left[\begin{array}{l}u \\ v\end{array}\right]\left(\cdot, u_{0}, v_{0}\right)$ from Theorem 3 originating at $\left[\begin{array}{l}u_{0} \\ v_{0}\end{array}\right] \in B$ exist and the $\operatorname{set}\left\{\left[\begin{array}{l}u \\ v\end{array}\right]\left(t, u_{0}, v_{0}\right):\left[\begin{array}{l}u_{0} \\ v_{0}\end{array}\right] \in B\right\}$ is bounded in $Y_{(\theta)}^{\zeta_{0}}$ for arbitrary $\zeta_{0} \in[0, \gamma(\varepsilon))$ and each $t \in\left(0, \tau_{B}\right)$. In particular, the set $\left\{\left[\begin{array}{l}u \\ v\end{array}\right]\left(t, u_{0}, v_{0}\right):\left[\begin{array}{l}u_{0} \\ v_{0}\end{array}\right] \in B\right\}$ is precompact in $Y_{(\theta)}^{\frac{n-2}{2(1-\theta)(n+2)}}$ for each $t \in\left(0, \tau_{B}\right)$.

Proof. The proof is a direct consequence of Remark 1, Theorem 3 (ii), and Theorem 4. 
In the considerations below, devoted to the existence of the global attractor to (2) in a subcritical case, we shall follow the general abstract scheme developed in $[\mathbf{1 0}, \mathbf{6}]$. For convenience we recall this scheme in the proposition below (see [10, Section 4.2]).

Proposition 2. Consider the Cauchy problem (14) where $P: D(P) \rightarrow Z$ is a sectorial, positive operator having compact resolvent, and $G: Z^{\alpha} \rightarrow Z$ is Lipschitz continuous on bounded subsets of $Z^{\alpha}$ for some $\alpha \in[0,1)$. Denote by $z\left(\cdot, z_{0}\right)$ a $Z^{\alpha}$-solution of (14) defined on a maximal interval of existence $\left[0, \tau_{z_{0}}\right)$. Then, the following two conditions are equivalent:

(i) Relation $S(t) z_{0}=z\left(t, z_{0}\right), t \geq 0$, defines on $Z^{\alpha}$ a compact $C^{0}$-semigroup $\{S(t)\}$ of global $Z^{\alpha}$-solutions to (14) which has a compact global attractor in $Z^{\alpha}$.

(ii) There are given:

- A Banach space $\mathcal{Y}$, with $D(P) \subset \mathcal{Y}$,

- a locally bounded function $C: \mathbb{R}^{+} \rightarrow \mathbb{R}^{+}$,

- a nondecreasing function $g: \mathbb{R}^{+} \longrightarrow \mathbb{R}^{+}$,

- a number $\gamma \in[0,1)$,

such that, for each $z_{0} \in Z^{\alpha}$, both

$$
\left\|z\left(t, z_{0}\right)\right\|_{\mathcal{Y}} \leq C\left(\left\|z_{0}\right\|_{Z^{\alpha}}\right), t \in\left(0, \tau_{z_{0}}\right),
$$

and

$$
\left\|G\left(z\left(t, z_{0}\right)\right)\right\|_{Z} \leq g\left(\left\|z\left(t, z_{0}\right)\right\|_{\mathcal{Y}}\right)\left(1+\left\|z\left(t, z_{0}\right)\right\|_{Z^{\alpha}}^{\gamma}\right), t \in\left(0, \tau_{z_{0}}\right),
$$

hold, and the estimate (19) is asymptotically independent of $z_{0} \in Z^{\alpha}$.

Based on the abstract scheme of Proposition 2 (ii) we shall prove the following theorem:

Theorem 5. Let (18) holds, $f$ satisfies (6) with $\rho \in\left(1, \frac{n+4(1-\theta)}{n-2}\right)$ and the dissipative Condition (7). Then, for the numbers $n, \theta$ admissible in (18) and $\alpha \in\left[\frac{n-2}{2(1-\theta)(n+2)}, 1\right)$ :

(i) There exists a compact $C^{0}$-semigroup $\left\{T_{(\theta), \alpha}(t)\right\}$ of global $Y_{(\theta)}^{\alpha}$-solutions to (2) which possesses a compact global attractor $\mathbf{A}_{(\theta), \alpha}$ in $Y_{(\theta)}^{\alpha}$,

(ii) $T_{(\theta), \alpha}(t)\left[\begin{array}{l}u_{0} \\ v_{0}\end{array}\right]=T_{(\theta), \frac{n-2}{2(1-\theta)(n+2)}}(t)\left[\begin{array}{l}u_{0} \\ v_{0}\end{array}\right]$, for $\left[\begin{array}{l}u_{0} \\ v_{0}\end{array}\right] \in Y_{(\theta)}^{\alpha}$ and $t \geq 0$; furthermore $\mathbf{A}_{(\theta), \alpha}=\mathbf{A}_{(\theta), \frac{n-2}{2(1-\theta)(n+2)}}=: \mathbf{A}_{(\theta)}$,

(iii) $T_{(\theta), 0}(t): Y^{0} \rightarrow Y_{(\theta)}^{\alpha}, \quad t>0$, where $T_{(\theta), 0}(t)\left[\begin{array}{l}u_{0} \\ v_{0}\end{array}\right]=\left[\begin{array}{l}u \\ v\end{array}\right]\left(t, u_{0}, v_{0}\right)$ and $\left[\begin{array}{l}u \\ v\end{array}\right]\left(\cdot, u_{0}, v_{0}\right)$ is an $\varepsilon$-regular solution from Theorem 3 , are well-defined maps being the extensions of $T_{(\theta), \alpha}(t), t>0$, to $Y^{0}$, 
(iv) $\mathbf{A}_{(\theta)}$ attracts bounded subsets of $Y^{0}$ under $\left\{T_{(\theta), \alpha}(t)\right\}$ in $Y_{(\theta)}^{\alpha}$-norm.

Proof. The Proof of (i) occurs in four steps.

Step 1 ( $Y^{0}$-estimate and the Lyapunov function). Take $\left[\begin{array}{l}u_{0} \\ v_{0}\end{array}\right] \in Y_{(\theta)}^{\alpha}$ and consider the corresponding $Y_{(\theta)}^{\alpha}$-solution $\left[\begin{array}{l}u \\ v\end{array}\right]\left(t, u_{0}, v_{0}\right)$ of $(2)$ resulting from Lemma 3. Multiply the equation for $v$ entering (2) by $v=\dot{u}$ in $L^{2}(\Omega)$ and use the properties of the negative Laplacian with Dirichlet boundary conditions to get

$$
\frac{d}{d t}\left(\frac{1}{2}\|v\|_{L^{2}(\Omega)}^{2}+\frac{1}{2}\left\|A^{\frac{1}{2}} u\right\|_{L^{2}(\Omega)}^{2}-\int_{\Omega} \int_{0}^{u} f(s) d s d x\right)=-\eta\left\|A^{\frac{\theta}{2}} v\right\|_{L^{2}(\Omega)}^{2} \leq 0 .
$$

This ensures in particular that

$$
\left\|\left[\begin{array}{l}
u \\
v
\end{array}\right]\left(t, u_{0}, v_{0}\right)\right\|_{Y^{0}} \leq \sqrt{c+c^{\prime} \mathcal{L}_{0}\left(\left[\begin{array}{l}
u_{0} \\
v_{0}
\end{array}\right]\right)} \leq C\left(\left\|\left[\begin{array}{l}
u_{0} \\
v_{0}
\end{array}\right]\right\|_{Y^{0}}\right),
$$

where $c, c^{\prime}$ do not depend on $\eta$,

$$
\begin{aligned}
\mathcal{L}_{0}\left(\left[\begin{array}{l}
w_{1} \\
w_{2}
\end{array}\right]\right)= & \frac{1}{2}\left\|w_{2}\right\|_{L^{2}(\Omega)}^{2}+\frac{1}{2}\left\|A^{\frac{1}{2}} w_{1}\right\|_{L^{2}(\Omega)}^{2} \\
& -\int_{\Omega} \int_{0}^{w_{1}} f(s) d s d x, \quad\left[\begin{array}{l}
w_{1} \\
w_{2}
\end{array}\right] \in Y^{0},
\end{aligned}
$$

and $C: \mathbb{R}^{+} \rightarrow \mathbb{R}^{+}$is a locally bounded function independent of $\eta$.

Step 2 (subordination of the nonlinearity to a power of $\left.\mathcal{A}_{(\theta)}\right)$. Since $1<\rho<$ $\frac{n+4(1-\theta)}{n-2}$, then based on the Nirenberg-Gagliardo type inequality we obtain that

$$
\begin{aligned}
& \left\|f\left(u\left(t, u_{0}, v_{0}\right)\right)\right\|_{L^{2}(\Omega)} \\
& \leq g\left(\left\|u\left(t, u_{0}, v_{0}\right)\right\|_{H^{1}(\Omega)}\right)\left(1+\left\|u\left(t, u_{0}, v_{0}\right)\right\|_{H^{1+2 \alpha_{1}(1-\theta)}(\Omega)}^{\gamma}\right),
\end{aligned}
$$

$t \in\left(0, \tau_{u_{0}, v_{0}}\right)$, with certain $\gamma \in[0,1), \alpha_{1} \in[0,1)$ and some nondecreasing function $g: \mathbb{R}^{+} \rightarrow \mathbb{R}^{+}$(see [10, Lemma 5.2.1]). Next, based on (23), we get the relation

$$
\begin{aligned}
& \left\|\mathcal{F}\left(\left[\begin{array}{l}
u \\
v
\end{array}\right]\left(t, u_{0}, v_{0}\right)\right)\right\|_{Y^{0}}=\left\|f\left(u\left(t, u_{0}, v_{0}\right)\right)\right\|_{L^{2}(\Omega)} \\
& \leq g\left(\left\|\left[\begin{array}{l}
u \\
v
\end{array}\right]\left(t, u_{0}, v_{0}\right)\right\|_{Y^{0}}\right)\left(1+\left\|\left[\begin{array}{l}
u \\
v
\end{array}\right]\left(t, u_{0}, v_{0}\right)\right\|_{Y_{(\theta)}^{\alpha_{1}}}^{\gamma}\right) .
\end{aligned}
$$


Step 3 (global solvability and compactness). Conditions (21) and (24) plus the compactness of the resolvent of $\mathcal{A}_{(\theta)}$ ensure that to (2) corresponds a compact $C^{0}$-semigroup $\left\{T_{(\theta), \alpha}(t)\right\}$ of global $Y_{(\theta)}^{\alpha}$-solutions having bounded orbits of bounded sets. For the proof of the existence of a global attractor for $\left\{T_{(\theta), \alpha}(t)\right\}$ in $Y_{(\theta)}^{\alpha}$ it now suffices to show that the estimate (21) is asymptotically independent of $\left[\begin{array}{l}u_{0} \\ v_{0}\end{array}\right] \in Y_{(\theta)}^{\alpha}$.

Step 4 (point dissipativeness of $\left\{T_{(\theta), \alpha}(t)\right\}$ - the role of the Lyapunov function). Functional $\mathcal{L}_{0}$ defined in (22) is a Lyapunov function for $\left\{T_{(\theta), \alpha}(t)\right\}$ in $Y_{(\theta)}^{\alpha}$. Therefore, $\omega$-limit sets of points from $Y_{(\theta)}^{\alpha}$ lie within the set $\mathcal{E}$ of all stationary solutions to (1). Our concern now is to prove that $\mathcal{E}$ is bounded in $Y^{0}$.

Let $\left[\begin{array}{c}\widetilde{u} \\ \widetilde{v}\end{array}\right] \in \mathcal{E}$. Then $\widetilde{v}=0$, whereas $\widetilde{u}$ is an $H^{2}(\Omega)$-solution of the elliptic problem

$$
\left\{\begin{array}{l}
-\Delta \widetilde{u}=f(\widetilde{u}), x \in \Omega, \\
\widetilde{u}=0 \text { on } \partial \Omega .
\end{array}\right.
$$

With the use of (7) it is easy to show that if $\widetilde{u}$ solves $(25)$, then $\|\widetilde{u}\|_{H^{1}(\Omega)} \leq c^{\prime \prime}$ where $c^{\prime \prime}=c^{\prime \prime}(\Omega, f)>0$ is independent of $\widetilde{u}$. Consequently, we have

$$
\left\|\left[\begin{array}{c}
\widetilde{u} \\
\widetilde{v}
\end{array}\right]\right\|_{Y^{0}} \leq c^{\prime \prime \prime},\left[\begin{array}{c}
\widetilde{u} \\
\widetilde{v}
\end{array}\right] \in \mathcal{E} .
$$

Since each $\omega$-limit set $\omega\left(\left[\begin{array}{l}u_{0} \\ v_{0}\end{array}\right]\right)$, lies in $\mathcal{E}$, is compact and attracts $\left[\begin{array}{l}u_{0} \\ v_{0}\end{array}\right] \in$ $Y_{(\theta)}^{\alpha}$ under $\left\{T_{(\theta), \alpha}(t)\right\}$ in $Y_{(\theta)}^{\alpha}$-norm, Condition (26) ensures in particular that

$$
\limsup _{t \rightarrow+\infty}\left\|\left[\begin{array}{l}
u \\
v
\end{array}\right]\left(t, u_{0}, v_{0}\right)\right\|_{Y^{0}} \leq c^{\prime \prime \prime},\left[\begin{array}{l}
u_{0} \\
v_{0}
\end{array}\right] \in Y_{(\theta)}^{\alpha} .
$$

Therefore, the estimate (21) is asymptotically independent of initial data from $Y_{(\theta)}^{\alpha}$ which completes the Proof of Assertion (i).

Part (ii) is a consequence of the smoothing action of $\left\{T_{(\theta), \alpha}(t)\right\}$. Part (iii) follows from Theorem 4. Finally, Part (iv) results from Lemma 4. Theorem 5 is thus proved.

In the above considerations we assumed (18) and required that $\rho$ in Theorem 5 is less than $\frac{n+4(1-\theta)}{n-2}$. These enabled the solutions to reach the space $Y_{(\theta)}^{1}$ and provided better control over the asymptotics.

Through the remaining part of this subsection let us assume merely that (6) holds with $\rho \in\left(1, \frac{n+2}{n-2}\right)$. If this is the case one may choose $\varepsilon>0$ 
arbitrarily small and satisfying $\rho<\frac{n+2}{n-2+4 \varepsilon(1-\theta)}$. This implies the inequality $\rho<\frac{n+2-4 \varepsilon \rho(1-\theta)}{n-2}$ and justifies that we take

$$
\rho \varepsilon<\gamma(\varepsilon)<\frac{n+2-\rho(n-2)}{4(1-\theta)} .
$$

Now, choosing $\varepsilon, \gamma(\varepsilon)$ according to (28) and defining $q=\frac{2 n}{n+2-4 \gamma(\varepsilon)(1-\theta)}$ we obtain the estimate

$$
\|u\|_{L^{\rho q}(\Omega)}^{\rho} \leq \text { const. }\|u\|_{H^{1}(\Omega)}^{\rho}
$$

and, consequently, we justify validity of the subordination condition

$$
\left\|\mathcal{F}\left(\left[\begin{array}{l}
u \\
v
\end{array}\right]\left(t, u_{0}, v_{0}\right)\right)\right\|_{Y_{(\theta)-1}^{\gamma(\varepsilon)}} \leq g\left(\left\|\left[\begin{array}{l}
u \\
v
\end{array}\right]\left(t, u_{0}, v_{0}\right)\right\|_{Y^{0}}\right)
$$

with an increasing function $g(s)=$ const.' $\left(1+s^{\rho}\right), s \geq 0$. Since the gap between $\gamma(\varepsilon)$ and $1+\varepsilon$ is less then 1, Lipschitz Condition (13) allows to obtain local $Y_{(\theta)}^{\varepsilon}$-solutions for (11) and apply the scheme of Proposition 2 with $Z^{0}=Y_{(\theta)_{-1}}^{\gamma(\varepsilon)}$ and $Z^{\alpha}:=Y_{(\theta)_{-1}}^{1+\varepsilon}=Y_{(\theta)}^{\varepsilon}$. Using similar arguments as in Steps 1-4 of Theorem 5 we thus obtain the following result.

Theorem 6. If $f$ satisfies (6) with $\rho \in\left(1, \frac{n+2}{n-2}\right)$ and the dissipativeness Condition (7) holds, then there exists $\varepsilon>0$ such that Conditions (i), (iii) and (iv) of Theorem 5 hold with $\alpha=\varepsilon$.

4.2. Subcritical case: $\theta=1$. In this section we restrict our attention to the case $\theta=1$ studied previously by many authors (see [15], [13], [11], $[16])$.

Remark 2. In the recent paper [16] the dimension of the global attractor was estimated. One can find however in this paper rather very strange errors. First, the author takes $X^{1} \times X^{1}$ as the domain of $\mathcal{A}_{(1)}$. However, if the base space is $Y^{0}$, this operator is not closed with such a domain. This is the case, when one needs to choose $Y_{(1)}^{1}$ as the domain of $\mathcal{A}_{(1)}$ following the description given in [9]. In this case it is thus rather unknown if the solution possesses the regularity stated in [16, Lemma 1 (ii)] for initial data from $Y^{0}$. Next in the proof of [16, Theorem 2] the author says that the semigroup $\left\{e^{-\mathcal{A}_{(1)} t}\right\}$ is compact. But this cannot be true because the resolvent of $\mathcal{A}_{(1)}$ is not compact. The latter may be easily seen if we look at the embeddings of $Y_{(1)}^{\alpha}$ spaces. Of course it is impossible for $Y_{(1)}^{\frac{1}{2}}=X^{\frac{1}{2}} \times X^{\frac{1}{2}}$ to be compactly embedded in $Y^{0}=X^{\frac{1}{2}} \times X^{0}$.

Throughout the present subsection we shall consider functions $f$ satisfying subcritical growth; that is (6) with $\rho<\frac{n+2}{n-2}$. In this particular case $\mathcal{F}$ 
takes $Y_{(1)_{-1}}^{1}$ into $Y_{(1)_{-1}}^{\frac{1}{2}}$ and is Lipschitz continuous in bounded sets (see [5, Lemma 3]). This says that the map $\mathcal{F}$ is subcritical and Theorem 3 can be rewritten in the following form.

Theorem 7. For any initial data $\left[\begin{array}{l}u_{0} \\ v_{0}\end{array}\right]$ lying in a bounded subset $B$ of $Y_{(1)_{-1}}^{1}$ there exists a number $\tau=\tau(B)$ and a unique 0 -regular solution $[0, \tau] \ni t \mapsto$ $\left[\begin{array}{l}u \\ v\end{array}\right]\left(t, u_{0}, v_{0}\right) \in Y_{(1)_{-1}}^{1}$ to (11) which depends continuously on the initial data and such that

$$
\left[\begin{array}{l}
u \\
v
\end{array}\right]\left(\cdot, u_{0}, v_{0}\right) \in C\left((0, \tau], Y_{(1)_{-1}}^{\frac{3}{2}}\right) \cap C^{1}\left((0, \tau], Y_{(1)_{-1}}^{\frac{3}{2}^{-}}\right) .
$$

Proof. The theorem above is a consequence of the results reported in [12].

We remark further that local solutions from Theorem 7 are bounded in the norm of $Y^{0}$ uniformly on bounded sets. As in the Proof of Theorem 5 one may show the estimate (29) below.

Lemma 5. Let $\left[\begin{array}{l}u \\ v\end{array}\right]\left(\cdot, u_{0}, v_{0}\right)$ be a solution obtained in Theorem 7 . If $f$ satisfies (6) with $\rho<\frac{n+2}{n-2}$ and the dissipativeness Condition (7), then

$$
\left\|\left[\begin{array}{l}
u \\
v
\end{array}\right]\left(t, u_{0}, v_{0}\right)\right\|_{Y^{0}} \leq c\left(\left[\begin{array}{l}
u_{0} \\
v_{0}
\end{array}\right]\right)
$$

where $c: R^{+} \rightarrow R^{+}$is a locally bounded function.

Our next step here is to prove that:

Lemma 6. Under the assumptions of Lemma 5 0-regular solutions from Theorem 7 exist globally in time and the problem (11) defines a $C^{0}$-semigroup $\left\{T_{(1), 0}(t)\right\}$ on $Y^{0}$ which has bounded orbits of bounded sets and is asymptotically smooth.

Proof. The existence of a $C^{0}$-semigroup with bounded orbits of bounded sets follows from Lemma 5 . To prove that $\left\{T_{(1), 0}(t)\right\}$ is asymptotically smooth we use the variation of constants formula

$$
T_{(1), 0}(t)\left[\begin{array}{l}
u_{0} \\
v_{0}
\end{array}\right]=e^{-\mathcal{A}_{(1)-1} t}\left[\begin{array}{l}
u_{0} \\
v_{0}
\end{array}\right]+\int_{0}^{t} e^{-\mathcal{A}_{(1)-1}(t-s)} \mathcal{F}\left(T_{(1), 0}(s)\left[\begin{array}{l}
u_{0} \\
v_{0}
\end{array}\right]\right) d s .
$$

Recall that $e^{-\mathcal{A}_{(1)}{ }_{-1} t}$ decays exponentially and that in the subcritical case $f$ takes bounded subsets of $X^{\frac{1}{2}}$ into bounded subsets of $X^{-\frac{1}{2}+\delta}$, for some $\delta>0$. From this we have that $\mathcal{F}$ is a compact map from $Y_{(1)_{-1}}^{1}=Y^{0}$ into 
$Y_{(1)_{-1}}^{\frac{1}{2}}=Y_{(1)_{-\frac{1}{2}}}$. Since $e^{-\mathcal{A}_{(1)_{-1}} t}$ is a bounded linear operator from $Y_{(1)_{-1}}^{\frac{1}{2}}$ to $Y_{(1)_{-1}}^{1}$ we have that the operator

$$
U(t)\left[\begin{array}{l}
u_{0} \\
v_{0}
\end{array}\right]=\int_{0}^{t} e^{-\mathcal{A}_{(1)-1}(t-s)} \mathcal{F}\left(T_{(1), 0}(s)\left[\begin{array}{l}
u_{0} \\
v_{0}
\end{array}\right]\right) d s
$$

as a map from $Y^{0}$ into $Y^{0}$ is compact. It follows from the results in [11] that $T_{(1), 0}(t)$ is asymptotically smooth as a sum of an exponentially decaying semigroup with a compact family of maps. This completes the proof.

As an immediate consequence of these lemmas and of Step 4 in Theorem 5 we have the following result.

Theorem 8. Under the assumptions of Lemma $5,\left\{T_{(1), 0}(t)\right\}$ has a compact global attractor $\mathbf{A}_{(1), 0}$ in $Y^{0}$.

The set $\mathbf{A}_{(1), 0}$ is bounded in $X^{\frac{1}{2}} \times X^{\frac{1}{2}}=Y_{(1)_{-1}}^{\frac{3}{2}}$ (see [10, Lemma 3.2.1]). Furthermore, noting that $\left\{T_{(1), 0}(t)\right\}$ is a dissipative $C^{0}$-semigroup in $Y_{(1)_{-1}}^{1}$ having bounded orbits of bounded sets, with simple computations based on the variation of constants formula one can easily see that $\left\{T_{(1), \alpha}(t)\right\}$ is a point dissipative $C^{0}$-semigroup in $Y_{(1)_{-1}}^{1+\alpha}$ with bounded orbits of bounded sets, for each $\alpha \in\left[0, \frac{1}{2}\right)$ (see [10, Corollary 4.3.2]). The semigroups $\left\{T_{(1), \alpha}(t)\right\}$ are also asymptotically smooth which follows as in Lemma 6 . This proves that:

Theorem 9. Under the assumptions of Lemma 5, the problem (11) defines a $C^{0}$-semigroup $\left\{T_{(1), \alpha}(t)\right\}$ on $Y_{(1)_{-1}}^{1+\alpha}$ which possesses a compact global attractor $\mathbf{A}_{(1), \alpha}$ for each $\alpha \in\left[0, \frac{1}{2}\right)$. Furthermore, $\mathbf{A}_{(1), 0}$ is bounded in $X^{\frac{1}{2}} \times X^{\frac{1}{2}}$ and $\mathbf{A}_{(1), \alpha}=\mathbf{A}_{(1), 0}$ for $\alpha \in\left[0, \frac{1}{2}\right)$.

4.3. Attractors in the critical growth case: $\theta=1$. In this subsection we shall consider the case when $f$ satisfies (6) with the critical exponent $\rho=\frac{n+2}{n-2}$.

4.3.1. The case of strong dissipation. We begin from the simpler case when the semigroup $\{T(t)\}$ corresponding to (11) is exponentially decaying and the attractor is a one point set $\{(0,0)\}$.

Proposition 3. Under the strong dissipative condition

$$
s f(s) \leq 0, s \in \mathbb{R},
$$

Equation (11) defines a $C^{0}$-semigroup on $Y^{0}$ which has a compact global attractor $\mathbf{A}_{(1), 0}=\{(0,0)\}$.

Proof. Note that both Theorem 7 and Lemma 5 remain true under the assumptions of the present subsection. Therefore, there exists corresponding 
to (11) semigroup $\left\{T_{(1), 0}(t)\right\}$ in $Y^{0}$ of global 0-regular solutions with bounded orbits of bounded sets. Based on (30) we shall next prove that

$$
\begin{aligned}
\left\|T_{(1), 0}(t)\left[\begin{array}{l}
u_{0} \\
v_{0}
\end{array}\right]\right\|_{Y^{0}}^{2} \leq h(r) e^{-M_{\widetilde{\delta}}(r) t}, \\
\qquad\left[\begin{array}{l}
u_{0} \\
v_{0}
\end{array}\right] \in B_{r}=B_{Y^{0}}\left(\left[\begin{array}{l}
0 \\
0
\end{array}\right], r\right), \quad t \geq 0, r>0,
\end{aligned}
$$

where $h(r)$ is described in (38) and $M_{\widetilde{\delta}}(r)$ is described in (34) and (36). In particular, $\{(0,0)\}$ is a unique equilibrium which attracts bounded subsets of $Y^{0}$.

Following [3] we introduce a functional

$$
\mathcal{L}_{\delta}\left(\left[\begin{array}{l}
w_{1} \\
w_{2}
\end{array}\right]\right)=\mathcal{L}_{0}\left(\left[\begin{array}{l}
w_{1} \\
w_{2}
\end{array}\right]\right)+\delta \int_{\Omega} w_{1} w_{2} d x, \delta \geq 0,\left[\begin{array}{l}
w_{1} \\
w_{2}
\end{array}\right] \in Y^{0}
$$

where $\mathcal{L}_{0}$ is a standard Lyapunov functional to (1) given in (22). We remark that as a consequence of (30) the integral $\int_{\Omega} \int_{0}^{w_{1}} f(s) d s d x$ is nonpositive. Therefore, for $\delta$ sufficiently small the quantity $\left\|\left[\begin{array}{l}w_{1} \\ w_{2}\end{array}\right]\right\|_{Y^{0}}^{2}$ is bounded by $8 \mathcal{L}_{\delta}\left(\left[\begin{array}{l}w_{1} \\ w_{2}\end{array}\right]\right)$ (see (37) below)

Estimating in a standard way we have

$$
\begin{aligned}
\frac{d}{d t} \mathcal{L}_{\delta}\left(T_{(1), 0}(t)\left[\begin{array}{l}
u_{0} \\
v_{0}
\end{array}\right]\right) \\
=-\eta\left\|A^{\frac{1}{2}} v\left(t, u_{0}, v_{0}\right)\right\|_{L^{2}(\Omega)}^{2}+\delta\left\|v\left(t, u_{0}, v_{0}\right)\right\|_{L^{2}(\Omega)}^{2} \\
\quad+\delta \int_{\Omega} u\left(t, u_{0}, v_{0}\right)\left(A u\left(t, u_{0}, v_{0}\right)+\eta A v\left(t, u_{0}, v_{0}\right)+f\left(u\left(t, u_{0}, v_{0}\right)\right)\right) d x \\
\leq-\frac{\eta}{2}\left\|A^{\frac{1}{2}} v\left(t, u_{0}, v_{0}\right)\right\|_{L^{2}(\Omega)}^{2}+\delta\left\|v\left(t, u_{0}, v_{0}\right)\right\|_{L^{2}(\Omega)}^{2} \\
\quad-\delta\left(1-\frac{\delta \eta}{2}\right)\left\|A^{\frac{1}{2}} u\left(t, u_{0}, v_{0}\right)\right\|_{L^{2}(\Omega)}^{2} .
\end{aligned}
$$

Applying next inequality $\|v\|_{L^{2}(\Omega)} \leq c_{\frac{1}{2}}\left\|A^{\frac{1}{2}} v\right\|_{L^{2}(\Omega)}$ we obtain for each $\delta \in$ $\left(0, \min \left\{\frac{\eta}{3 c_{\frac{1}{2}}^{2}}, \frac{1}{\eta}\right\}\right)$ the estimate

$$
\frac{d}{d t} \mathcal{L}_{\delta}\left(T_{(1), 0}(t)\left[\begin{array}{l}
u_{0} \\
v_{0}
\end{array}\right]\right) \leq-\frac{\delta}{2}\left\|v\left(t, u_{0}, v_{0}\right)\right\|_{L^{2}(\Omega)}^{2}-\frac{\delta}{2}\left\|A^{\frac{1}{2}} u\left(t, u_{0}, v_{0}\right)\right\|_{L^{2}(\Omega)}^{2} .
$$


Since (30) implies in particular that $f(0)=0$, Condition (6) ensures that

$$
\begin{aligned}
& \exists_{\widetilde{c} \geq 1}\left|\int_{\Omega} \int_{0}^{w_{1}} f(s) d s d x\right| \\
& \leq \widetilde{c}\left(1+\left\|A^{\frac{1}{2}} w_{1}\right\|_{L^{2}(\Omega)}^{\frac{4}{n-2}}\right)\left\|A^{\frac{1}{2}} w_{1}\right\|_{L^{2}(\Omega)}^{2}, \quad w_{1} \in H_{0}^{1}(\Omega) .
\end{aligned}
$$

\section{Defining}

$$
\begin{aligned}
& \text { (34) } M_{r}=\sup \left\{\left\|A^{\frac{1}{2}} u\left(t, u_{0}, v_{0}\right)\right\|_{L^{2}(\Omega)}^{\frac{4}{n-2}} ;\left[\begin{array}{l}
u_{0} \\
v_{0}
\end{array}\right] \in B_{r}, t \geq 0\right\}, \\
& M_{\delta}(r)=\frac{\delta}{8 \widetilde{c}\left(1+M_{r}^{\frac{4}{n-2}}\right)}, \text { where } \widetilde{c} \geq 1 \text { and } \delta \in\left(0, \min \left\{\frac{\eta}{3 c_{\frac{1}{2}}^{2}}, \frac{1}{\eta}\right\}\right),
\end{aligned}
$$

we may increase the right-hand side of (33) to get

$$
\begin{aligned}
& \frac{d}{d t} \mathcal{L}_{\delta}\left(T_{(1), 0}(t)\left[\begin{array}{l}
u_{0} \\
v_{0}
\end{array}\right]\right) \\
& \leq-M_{\delta}(r) \mathcal{L}_{\delta}\left(T_{(1), 0}(t)\left[\begin{array}{l}
u_{0} \\
v_{0}
\end{array}\right]\right)-M_{\delta}(r) \int_{\Omega} \int_{0}^{u\left(t, u_{0}, v_{0}\right)} f(s) d s d x \\
& \quad+\delta M_{\delta}(r) \int_{\Omega} u\left(t, u_{0}, v_{0}\right) v\left(t, u_{0}, v_{0}\right) d x-\frac{\delta}{4}\left\|v\left(t, u_{0}, v_{0}\right)\right\|_{L^{2}(\Omega)}^{2} \\
& \quad-\frac{\delta}{4}\left\|A^{\frac{1}{2}} u\left(t, u_{0}, v_{0}\right)\right\|_{L^{2}(\Omega)}^{2} \\
& \leq-M_{\delta}(r) \mathcal{L}_{\delta}\left(T_{(1), 0}(t)\left[\begin{array}{l}
u_{0} \\
v_{0}
\end{array}\right]\right)+\delta\left(\frac{c_{\frac{1}{2}}^{2} M_{\delta}(r)}{2}-\frac{1}{8}\right)\left\|A^{\frac{1}{2}} u\left(t, u_{0}, v_{0}\right)\right\|_{L^{2}(\Omega)}^{2} \\
& \quad+\delta\left(\frac{M_{\delta}(r)}{2}-\frac{1}{4}\right)\left\|v\left(t, u_{0}, v_{0}\right)\right\|_{L^{2}(\Omega)}^{2}, \\
& \quad \delta \in\left(0, \min \left\{\frac{\eta}{3 c_{\frac{1}{2}}^{2}}, \frac{1}{\eta}\right\}\right),\left[\begin{array}{l}
u_{0} \\
v_{0}
\end{array}\right] \in B_{r}, t \geq 0 .
\end{aligned}
$$

Let us next choose $\delta=\widetilde{\delta}$ so small that both

(36) $\widetilde{\delta}\left(\frac{c_{\frac{1}{2}}^{2} M_{\widetilde{\delta}}(r)}{2}-\frac{1}{8}\right)\left\|A^{\frac{1}{2}} u\left(t, u_{0}, v_{0}\right)\right\|_{L^{2}(\Omega)}^{2}$

$$
+\widetilde{\delta}\left(\frac{M_{\widetilde{\delta}}(r)}{2}-\frac{\widetilde{\delta}}{4}\right)\left\|v\left(t, u_{0}, v_{0}\right)\right\|_{L^{2}(\Omega)}^{2} \leq 0
$$


and

$$
\frac{1}{8}\left\|T_{(1), 0}(t)\left[\begin{array}{l}
u_{0} \\
v_{0}
\end{array}\right]\right\|_{Y^{0}}^{2} \leq \mathcal{L}_{\widetilde{\delta}}\left(T_{(1), 0}(t)\left[\begin{array}{l}
u_{0} \\
v_{0}
\end{array}\right]\right)
$$

are satisfied. For such value of $\delta$ inequality (35) reads:

$$
\frac{d}{d t} \mathcal{L}_{\widetilde{\delta}}\left(T_{(1), 0}(t)\left[\begin{array}{l}
u_{0} \\
v_{0}
\end{array}\right]\right) \leq-M_{\widetilde{\delta}}(r) \mathcal{L}_{\widetilde{\delta}}\left(T_{(1), 0}(t)\left[\begin{array}{l}
u_{0} \\
v_{0}
\end{array}\right]\right),\left[\begin{array}{l}
u_{0} \\
v_{0}
\end{array}\right] \in B_{r}, t \geq 0
$$

and we obtain the estimate

$$
\left\|T_{(1), 0}(t)\left[\begin{array}{l}
u_{0} \\
v_{0}
\end{array}\right]\right\|_{Y^{0}}^{2} \leq 8 \mathcal{L}_{\widetilde{\delta}}\left(T_{(1), 0}(t)\left[\begin{array}{l}
u_{0} \\
v_{0}
\end{array}\right]\right) \leq h(r) e^{-M_{\widetilde{\delta}}(r) t},\left[\begin{array}{l}
u_{0} \\
v_{0}
\end{array}\right] \in B_{r}, t \geq 0
$$

where

$$
h(r)=8 \sup \left\{\mathcal{L}_{\widetilde{\delta}}\left(\left[\begin{array}{l}
u_{0} \\
v_{0}
\end{array}\right]\right) ;\left[\begin{array}{l}
u_{0} \\
v_{0}
\end{array}\right] \in B_{r}\right\} .
$$

The proof is complete.

4.3.2. Nonlinear variation of constants formula. Our next concern is to prove for a pair of problems (39), (40) given below the Alekseev's nonlinear variation of constants formula (42) (see [3, Theorem 2.2]). In these considerations we shall need the following assumptions:

$\left(H_{0}\right) P$ is a sectorial, positive operator in a Banach space $Z=Z^{0}$ with the domain $Z^{1}$ and for some $\alpha \in[0,1)$ functions $G_{1}: Z^{\alpha} \rightarrow Z^{0}$, $G_{2}: Z^{\alpha} \rightarrow Z^{\alpha}$ are such that $G_{1}$ has continuous Frechét derivative and $G_{2}$ is Lipschitz continuous on bounded sets.

$\left(H_{1}\right)$ There exists a Banach space $\mathcal{Y}=\mathcal{Y}^{0}$ densely embedded in $Z^{\alpha}$ such that $P_{\left.\right|_{\mathcal{y}^{0}}}\left(P_{\left.\right|_{\mathcal{Y}} 0}\right.$ being a realization of $P$ in $\left.Y^{0}\right)$ is sectorial and positive in $\mathcal{Y}^{0}$ with the domain $\mathcal{Y}^{1}$ and $G_{1}, G_{2}$ are Lipschitz continuous on bounded sets as the maps from $\mathcal{Y}^{0}$ into $\mathcal{Y}^{0}$.

For $\xi \in Z^{\alpha}$ let $z=z(t, \xi)$ be a solution (as in [12, Chapter 3]) to

$$
\dot{z}+P z=G_{1}(z), t>0, z(0)=\xi .
$$

Similarly, let $\widetilde{z}=\widetilde{z}(t, \xi)$ be a solution to

$$
\dot{\widetilde{z}}+P \widetilde{z}=G_{1}(\widetilde{z})+G_{2}(\widetilde{z}), t>0, \widetilde{z}(0)=\xi .
$$

Lemma 7. Suppose that the requirements of $\left(H_{0}\right)$ and $\left(H_{1}\right)$ are satisfied. Then, the following conditions hold:

$$
\text { The function }(0,+\infty) \times Z^{\alpha} \ni(t, \omega) \rightarrow z(t, \omega) \in Z^{\alpha}
$$
has continuous Frechét derivative,

$$
\widetilde{z}(t, \xi)=z(t, \xi)+\int_{0}^{t} \frac{\partial z}{\partial \omega}(t-s, \widetilde{z}(s, \xi)) G_{2}(\widetilde{z}(s, \xi)) d s, t>0 .
$$


Proof. Condition (41) is a consequence of [12, Corollary 3.4.6]. Next, since $z(t, \omega)$ in $(41)$ is a $C^{1}$-function, using the chain rule we obtain

$$
\frac{d}{d s}[z(t-s, \widetilde{z}(s, \xi))]=-\dot{z}(t-s, \widetilde{z}(s, \xi))+\frac{\partial z}{\partial \omega}(t-s, \widetilde{z}(s, \xi)) \dot{\widetilde{z}}(s, \xi) .
$$

For $\xi \in \mathcal{Y}^{0}$, assumptions of $\left(H_{1}\right)$ guarantee that $\widetilde{z}(s, \xi) \in \mathcal{Y}^{1}$ and $\dot{z}(0, \widetilde{z}(s, \xi))$ exists in $\mathcal{Y}^{0}$-norm. Since $\mathcal{Y}^{0} \subset Z^{\alpha}$, the derivative $\dot{z}(0, \widetilde{z}(s, \xi))$ exists in $Z^{\alpha}$ norm and we have:

$$
\begin{aligned}
\dot{z}(t-s, \widetilde{z}(s, \xi)) & =\lim _{h \rightarrow 0^{+}} \frac{z(t-s+h, \widetilde{z}(s, \xi))-z(t-s, \widetilde{z}(s, \xi))}{h} \\
& =\lim _{h \rightarrow 0^{+}} \frac{z(t-s, z(h, \widetilde{z}(s, \xi)))-z(t-s, z(0, \widetilde{z}(s, \xi)))}{h} \\
& =\frac{\partial z}{\partial \omega}(t-s, \widetilde{z}(s, \xi)) \dot{z}(0, \widetilde{z}(s, \xi)) .
\end{aligned}
$$

Connecting (43), (44), and (40) we get

$$
\begin{aligned}
\frac{d}{d s}[z(t-s, \widetilde{z}(s, \xi))]= & \frac{\partial z}{\partial \omega}(t-s, \widetilde{z}(s, \xi))\left(P z(0, \widetilde{z}(s, \xi))-G_{1}(z(0, \widetilde{z}(s, \xi)))\right. \\
& \left.-P \widetilde{z}(s, \xi)+G_{1}(\widetilde{z}(s, \xi))+G_{2}(\widetilde{z}(s, \xi))\right) \\
= & \frac{\partial z}{\partial \omega}(t-s, \widetilde{z}(s, \xi)) G_{2}(\widetilde{z}(s, \xi)) .
\end{aligned}
$$

Integrating both sides of (45) we show that (42) holds for $\xi \in \mathcal{Y}^{0}$.

Now choose $\xi_{0} \in Z^{\alpha}$ and consider a sequence $\left\{\xi_{n}\right\} \in \mathcal{Y}^{0}$ convergent to $\xi_{0}$ in $Z^{\alpha}$. We know that

$$
\widetilde{z}\left(t, \xi_{n}\right)=z\left(t, \xi_{n}\right)+\int_{0}^{t} \frac{\partial z}{\partial \omega}\left(t-s, \widetilde{z}\left(s, \xi_{n}\right)\right) G_{2}\left(\widetilde{z}\left(s, \xi_{n}\right)\right) d s, t>0, n \in \mathbb{N},
$$

where $z\left(\cdot, \xi_{n}\right)$ and $\widetilde{z}\left(\cdot, \xi_{n}\right)$ tend in $Z^{\alpha}$ to $z\left(\cdot, \xi_{0}\right)$ and $\widetilde{z}\left(\cdot, \xi_{0}\right)$ respectively. Since convergence of $z\left(\cdot, \xi_{n}\right)$ and $\widetilde{z}\left(\cdot, \xi_{n}\right)$ is uniform with respect to $t$ varying in compact subintervals of $[0,+\infty)$ (see [12, Theorem 3.4.1]), passing to the limit in (46) we obtain (42) for $\xi \in Z^{\alpha}$. The proof is complete.

Remark 3. Lemma 7 remains true if instead of $\left(H_{0}\right)$ and $\left(H_{1}\right)$ we assume that $\left(H_{0}^{\prime}\right)$ and $\left(H_{1}\right)$ hold.

$\left(H_{0}^{\prime}\right) P$ is a sectorial, positive operator in a Banach space $Z=Z^{0}$ with the domain $Z^{1}, \alpha \geq \beta \geq 0$ satisfy $\alpha-\beta \in[0,1)$ and functions $G_{1}: Z^{\alpha} \rightarrow$ $Z^{\beta}, G_{2}: Z^{\alpha} \rightarrow Z^{\alpha}$ are such that $G_{1}$ has continuous Frechét derivative and $G_{2}$ is Lipschitz continuous on bounded sets.

Proof. Indeed, since $P_{Z^{\beta}}\left(P_{Z^{\beta}}\right.$ being the realization of $P$ in $\left.W^{0}:=Z^{\beta}\right)$ is a sectorial, positive operator with $D\left(P_{Z^{\beta}}\right)=Z^{\beta+1}=$ : $W^{1}$ (see $[\mathbf{1 0}$, 
Proposition 1.3.8]) and, for $\alpha^{\prime}=\alpha-\beta, W^{\alpha^{\prime}}=\left(Z^{\beta}\right)^{\alpha-\beta}=Z^{\alpha}$, (see [1, p. 260]) we repeat the arguments of Lemma 7 with $P_{Z^{\beta}}, W^{0}$ and $W^{\alpha}$ instead of $P, Z^{0}, Z^{\alpha}$.

The next lemma shows validity of the Alekseev's formula for a pair of sectorial problems (see (47), (48) below) connected to the strongly damped wave Equation (1).

Lemma 8. Let $n=3,4,5,6$. Suppose that:

- $f=f_{1}+f_{2}, f_{i}: \mathbb{R} \rightarrow \mathbb{R}, i=1,2$,

- $f_{1}$ satisfies (6) with $\rho=\frac{n+2}{n-2}, f_{1}$ has second order derivative, $\left|f_{1}^{\prime \prime}(s)\right| \leq$ $c\left(1+|s|^{\frac{n+2}{n-2}-2}\right)$, and, in addition, $s f_{1}(s) \leq 0$ for $s \in \mathbb{R}$,

- $f_{2}$ satisfies (6) with $\rho \leq \frac{n}{n-2}$ and, moreover, $\lim _{\sup } \operatorname{su}_{|s| \rightarrow \infty} \frac{f_{2}(s)}{s} \leq 0$.

Then:

(i) Assumptions of $\left(H_{0}^{\prime}\right)$ hold with $P=\mathcal{A}_{(1)_{-1}}, Z^{0}=Y_{(1)_{-1}}, \alpha=1, \beta=\frac{1}{2}$, and

$$
G_{1}\left(\left[\begin{array}{l}
w_{1} \\
w_{2}
\end{array}\right]\right)=\left[\begin{array}{c}
0 \\
F_{1}\left(w_{1}\right)
\end{array}\right], G_{2}\left(\left[\begin{array}{l}
w_{1} \\
w_{2}
\end{array}\right]\right)=\left[\begin{array}{c}
0 \\
F_{2}\left(w_{1}\right)
\end{array}\right],
$$

where $F_{1}, F_{2}$ are Nemitskiน maps corresponding to $f_{1}$ and $f_{2}$ respectively.

(ii) Assumptions of $\left(H_{1}\right)$ hold with $\alpha=1, \mathcal{Y}^{0}=X^{1} \times X^{0}, \mathcal{Y}^{1}=X^{1} \times X^{1}$, and $P_{\left.\right|_{\mathcal{Y}^{0}}}=\mathcal{A}_{(1)_{X^{1} \times X^{0}}}$.

(iii) Alekseev's formula (42) holds with $\xi:=\left[\begin{array}{l}u_{0} \\ v_{0}\end{array}\right], \widetilde{z}:=T(\cdot)\left[\begin{array}{l}u_{0} \\ v_{0}\end{array}\right]$ denoting the solution to

$$
\begin{aligned}
& \frac{d}{d t}\left[\begin{array}{l}
u \\
v
\end{array}\right]+\mathcal{A}_{(1)_{-1}}\left[\begin{array}{l}
u \\
v
\end{array}\right]=\left[\begin{array}{c}
0 \\
F_{1}(u)
\end{array}\right]+\left[\begin{array}{c}
0 \\
F_{2}(u)
\end{array}\right], t>0,\left[\begin{array}{l}
u \\
v
\end{array}\right]_{t=0}=\left[\begin{array}{l}
u_{0} \\
v_{0}
\end{array}\right], \\
& \text { and } z:=S\left(\cdot,\left[\begin{array}{l}
u_{0} \\
v_{0}
\end{array}\right]\right) \text { denoting the solution to }
\end{aligned}
$$

$$
\frac{d}{d t}\left[\begin{array}{l}
u \\
v
\end{array}\right]+\mathcal{A}_{(1)_{-1}}\left[\begin{array}{l}
u \\
v
\end{array}\right]=\left[\begin{array}{c}
0 \\
F_{1}(u)
\end{array}\right], t>0,\left[\begin{array}{l}
u \\
v
\end{array}\right]_{t=0}=\left[\begin{array}{l}
u_{0} \\
v_{0}
\end{array}\right] .
$$

(iv) $S\left(t,\left[\begin{array}{l}u_{0} \\ v_{0}\end{array}\right]\right)$ exponentially decays to 0 , uniformly for $\left[\begin{array}{l}u_{0} \\ v_{0}\end{array}\right]$ varying in bounded subsets of $Y^{0}$.

Proof. The Proof of (i) is standard. For the validity of (ii) the crucial property is that $\mathcal{A}_{\left.(1)\right|_{X^{1} \times X^{0}}}$ defines a sectorial operator (see [15, Proposition 2.2]). Condition (iii) follows from Lemma 7. Finally, the convergence in (iv) is a consequence of Proposition 3. 
4.3.3. Existence theorem. With the use of Alekseev's formula we may finally obtain the existence of a compact global attractor for the semigroup $\{T(t)\}$ corresponding to (1) in the critical growth case.

Theorem 10. Under the assumptions of Lemma 8 and the additional assumption that $f_{2}$ is continuously differentiable function with $\left|f_{2}^{\prime}\right| \leq c$, the problem (47) defines in $Y^{0}$ a $C^{0}$-semigroup $\{T(t)\}$ of 0 -regular solutions which possesses a compact global attractor in $Y^{0}$.

Proof. The assertions of Theorem 7 and Lemma 5 remain valid under the assumptions of the present theorem. The existence of a $C^{0}$-semigroup $\{T(t)\}$ in $Y^{0}$ with bounded orbits of bounded sets is thus straightforward. If we proved that $\{T(t)\}$ is asymptotically smooth, then the existence of a Lyapunov functional $\mathcal{L}_{0}$ (see (22)) and the boundedness of the set of stationary solutions would guarantee that $\{T(t)\}$ is point dissipative. Consequently, $\{T(t)\}$ would possess a compact global attractor in $Y^{0}$.

To prove that $\{T(t)\}$ is asymptotically smooth we apply Lemma 8 (iii) decomposing $\{T(t)\}$ so that

$$
T(t)\left[\begin{array}{l}
u_{0} \\
v_{0}
\end{array}\right]=S\left(t,\left[\begin{array}{l}
u_{0} \\
v_{0}
\end{array}\right]\right)+U(t)\left[\begin{array}{l}
u_{0} \\
v_{0}
\end{array}\right],\left[\begin{array}{l}
u_{0} \\
v_{0}
\end{array}\right] \in Y^{0}, t \geq 0
$$

where $S\left(\cdot,\left[\begin{array}{l}u_{0} \\ v_{0}\end{array}\right]\right)$ is a solution to $(48)$ and

$$
U(t)\left[\begin{array}{l}
u_{0} \\
v_{0}
\end{array}\right]=\int_{0}^{t} \frac{\partial S}{\partial \omega}\left(t-s, T(s)\left[\begin{array}{l}
u_{0} \\
u_{0}
\end{array}\right]\right) G_{2}\left(T(s)\left[\begin{array}{l}
u_{0} \\
v_{0}
\end{array}\right]\right) d s .
$$

By Lemma 8 (iv) to justify asymptotic smoothness of $\{T(t)\}$ we only need to prove that $U(t): Y^{0} \rightarrow Y^{0}$ is a compact map for each $t>0$ (see $[\mathbf{1 1}$, Lemma 3.2.3]).

As a consequence of the growth restriction for $f_{2}, F_{2}$ takes bounded subsets of $X^{\frac{1}{2}}$ into bounded subsets of $X^{\delta}$ for any $\delta \in\left(0, \frac{1}{4}\right)$ (see [3, Lemma 5.2]). This suggests that it might be possible to take advantage of the smoothing properties of analytic semigroups and study the equation for $\frac{\partial S}{\partial \omega}\left(t-s, T(s)\left[\begin{array}{l}u_{0} \\ u_{0}\end{array}\right]\right) G_{2}\left(T(s)\left[\begin{array}{l}u_{0} \\ v_{0}\end{array}\right]\right)$ in another space which would be slightly smoother than the original base space $Y^{0}$.

For this purpose fix certain $\delta_{0} \in\left(0, \frac{1}{4}\right)$ and set $E^{0}=E=X^{\frac{1}{2}+\delta_{0}} \times X^{\delta_{0}}$. Then $\mathcal{A}_{(1)}=\mathcal{A}_{(1)_{\mid} E^{0}}$ is sectorial on $E^{0}$ (see [13, Theorem 1.1]) and we verify (similarly as in $\left[\mathbf{5}\right.$, Proposition 1 and Lemma 1]) that $\mathcal{A}_{(1)}=\mathcal{A}_{(1)_{\mid}{ }_{E^{0}}}$ considered on a base space $E^{0}$ with the domain $E^{1}=\left\{\left[\begin{array}{c}\phi \\ \psi\end{array}\right] \in X^{\frac{1}{2}+\delta_{0}} \times\right.$ $\left.X^{\frac{1}{2}+\delta_{0}}: \phi+\eta \psi \in X^{1+\delta_{0}}\right\}$ is maximal accretive with zero in the resolvent set. 
Generally speaking, it may be observed that a number of facts previously proved for $\mathcal{A}_{(1)}$ defined on $Y^{0}$ with the aid of $A: X^{1} \rightarrow X$, may be reproved for $\mathcal{A}_{(1)}=\mathcal{A}_{(1)_{\mid}{ }_{E} 0}$, that is for $\mathcal{A}_{(1)}$ redefined on $E^{0}$ with the aid of $A_{\left.\right|_{X} \delta_{0}}$ : $X^{1+\delta_{0}} \rightarrow X^{\delta_{0}}$ instead of $A: X^{1} \rightarrow X$ (see (3));

$$
\mathcal{A}_{(1)_{\mid} E^{0}}\left[\begin{array}{c}
\varphi \\
\psi
\end{array}\right]=\left[\begin{array}{c}
-\psi \\
A_{\left.\right|_{X^{\delta_{0}}}}(\varphi+\eta \psi)
\end{array}\right]=\mathcal{A}_{(1)}\left[\begin{array}{l}
\varphi \\
\psi
\end{array}\right] \text { for }\left[\begin{array}{l}
\varphi \\
\psi
\end{array}\right] \in E^{1}
$$

For example, since $A_{\left.\right|_{X} \delta_{0}}$ is selfadjoint and positive definite on $E^{0}$, we may use the general results of $[\mathbf{9}]$ to get the characterization

$$
\begin{aligned}
E^{\alpha} & =D\left(\left(A_{\left.\right|_{X} \delta_{0}}\right)^{\frac{1}{2}}\right) \times D\left(\left(A_{\left.\right|_{X} \delta_{0}}\right)^{\alpha}\right)=\left[X^{\delta_{0}}, X^{1+\delta_{0}}\right]_{\frac{1}{2}} \times\left[X^{\delta_{0}}, X^{1+\delta_{0}}\right]_{\alpha} \\
& =X^{\frac{1}{2}+\delta_{0}} \times X^{\alpha+\delta_{0}}, \alpha \in\left[0, \frac{1}{2}\right] .
\end{aligned}
$$

In particular $E^{\frac{1}{2}}$ is thus a product space $X^{\frac{1}{2}+\delta_{0}} \times X^{\frac{1}{2}+\delta_{0}}$, so that repeating part of the proof of [5, Lemma 1] we get the inclusion

$$
E_{-1}^{\frac{1}{2}}=E_{-\frac{1}{2}} \supset X^{\frac{1}{2}+\delta_{0}} \times X^{-\frac{1}{2}+\delta_{0}} .
$$

By our assumptions, $F_{1}: X^{\frac{1}{2}} \rightarrow X^{-\frac{1}{2}}$ and consequently also $G_{1}: Y_{(1)_{-1}}^{1} \rightarrow$ $Y_{(1)_{-1}}^{\frac{1}{2}}$ are Frechét differentiable functions and

$$
\begin{aligned}
S=S\left(\cdot, V_{0}\right)= & {\left[\begin{array}{c}
w \\
\dot{w}
\end{array}\right], } \\
& \text { where } V_{0} \in\left\{T(s)\left[\begin{array}{l}
u_{0} \\
v_{0}
\end{array}\right]: s>0,\left\|\left[\begin{array}{l}
u_{0} \\
v_{0}
\end{array}\right]\right\|_{Y^{0}} \leq r\right\}=: \Sigma_{r},
\end{aligned}
$$

fulfills the relations

$$
\frac{d S}{d t}\left(t, V_{0}\right)+\mathcal{A}_{(1)_{-1}} S\left(t, V_{0}\right)=G_{1}\left(S\left(t, V_{0}\right)\right), t>0, S\left(0, V_{0}\right)=V_{0} .
$$

Consequently, from $\left[\mathbf{1 2}\right.$, Theorem 3.4.4] we know that $V=\frac{\partial S}{\partial \omega}\left(\cdot, V_{0}\right) G_{2}\left(V_{0}\right)$ is a mild solution in $Y_{(1)_{-1}}^{1}$ of the equation

$$
\frac{d \mathcal{W}}{d t}+\mathcal{A}_{(1)_{-1}} \mathcal{W}=G_{1}^{\prime}\left(S\left(t, V_{0}\right)\right) \mathcal{W}, t>0
$$

where

$\mathcal{W}=\left[\begin{array}{c}\chi \\ \dot{\chi}\end{array}\right], G_{1}^{\prime}\left(S\left(t, V_{0}\right)\right) \mathcal{W}=\left[\begin{array}{c}0 \\ f_{1}^{\prime}(w(t)) \chi\end{array}\right]=: \mathcal{H}(t, \mathcal{W})$ and $\mathcal{W}(0)=G_{2}\left(V_{0}\right)$ 
Equation (49) has a uniqueness property since $\mathcal{H}: R^{+} \times Y^{0} \rightarrow Y_{(1)_{-1}}^{\frac{1}{2}}$ is Hölder continuous with respect to the first argument and Lipschitz continuous with respect to the second argument uniformly on bounded subsets of $R^{+} \times E^{0}$.

If we justified that the equation

$$
\frac{d \mathcal{W}}{d t}+\left(\mathcal{A}_{(1)_{E^{0}}}\right)_{-1} \mathcal{W}=G_{1}^{\prime}\left(S\left(t, V_{0}\right)\right) \mathcal{W}, t>0, \mathcal{W}(0)=G_{2}\left(V_{0}\right),
$$

has a unique solution in $E^{0}$, it would have to coincide with $V$ and we could study $V$ as the solution to (50). If we could additionally estimate the solution to (50) in $E_{0}$, we would be able finally to justify compactness of $U(t): Y^{0} \rightarrow$ $Y^{0}$ using the fact that bounded subsets of $E^{0}$ are precompact in $Y^{0}$.

For the solvability of (50) with the initial data in $E^{0}$ it suffices to justify that $\mathcal{H}: R^{+} \times E^{0} \rightarrow E_{-1}^{\frac{1}{2}}$ is Hölder continuous with respect to the first argument and Lipschitz continuous with respect to the second argument uniformly on bounded subsets of $R^{+} \times E^{0}$. We remark, omitting detailed calculations, that the Hölder continuity follows from the fact that (since $V_{0}$ belongs to $\left.Y_{(1)}^{\frac{1}{2}}\right) S\left(\cdot, V_{0}\right): R^{+} \rightarrow Y^{0}$ is Hölder continuous on bounded sets as well as from the existence of $f^{\prime \prime}$ with the prescribed growth. The Lipschitz continuity is a result of the growth restriction for $f^{\prime}$.

We next find the estimate

$$
\left\|f_{1}^{\prime}(w(t)) \chi\right\|_{X^{-\frac{1}{2}+\delta_{0}}} \leq h\left(\|w(t)\|_{X^{\frac{1}{2}}}\right)\left(1+\|\chi\|_{X^{\frac{1}{2}+\delta_{0}}}\right), \chi \in X^{\frac{1}{2}+\delta_{0}}, t \geq 0,
$$

(here $h: R^{+} \rightarrow R^{+}$is a nondecreasing function) which shows that $V$ is the global solution in $E^{0}$ to the problem (50), where the growth of nonlinear term $\mathcal{H}(t, \mathcal{W})$ is sublinear, i.e.,

$$
\|\mathcal{H}(t, \mathcal{W})\|_{E_{-1}^{\frac{1}{2}}} \leq h\left(\left\|S\left(t, V_{0}\right)\right\|_{Y^{0}}\right)\left(1+\|\mathcal{W}(t)\|_{E^{0}}\right), t>0 .
$$

Since $G_{2}\left(V_{0}\right) \in E^{0}$ and since $\left\|G_{2}\left(V_{0}\right)\right\|_{E^{0}} \leq C\left(\left\|V_{0}\right\|_{Y^{0}}\right)$ for some continuous function $C(\cdot)$ and since both $\{T(t)\}$ (which controls $V_{0}$ ) and $\{S(t)\}$ (which enters the crucial estimate (51)) have orbits of bounded subsets of $Y^{0}$ bounded in the norm of $Y^{0}$, after standard calculations based on the integral counterpart of (50) we obtain that

$$
\|\mathcal{W}(t)\|_{E^{0}} \leq \text { const. }(r, \tau), t \in[0, \tau], V_{0} \in \Sigma_{r}, \tau \geq 0, r>0 .
$$

What was said above makes clear that we may substitute $\mathcal{W}=V$ into the left-hand side of (52). Compactness of $U(t)$ follows thus from (52) and from compactness of the embedding $E^{0} \subset Y^{0}$. The Proof of Theorem 10 is complete. 
Acknowledgment. This work was carried out while the second author visited the Department of Mathematics of the Instituto de Ciências Matemáticas e de Computação, Universidade de São Paulo, Brazil. He would like to acknowledge the great hospitality of the people from this Institution.

\section{References}

[1] H. Amann, Linear and Quasilinear Parabolic Problems, Birkhäuser, Basel, 1995, MR 96g:34088, Zbl 0819.35001.

[2] J.M. Arietta and A.N. Carvalho, Abstract parabolic problems with critical nonlinearities and applications to Navier-Stokes and heat equations, Trans. Amer. Math. Soc., 352 (2000), 285-310, MR 2000i:34115, Zbl 0940.35119.

[3] J.M. Arietta, A.N. Carvalho and J.K. Hale, A damped hyperbolic equation with critical exponent, Commun. in Partial Differential Equations, 17(5-6) (1992), 841-866, MR 93f:35145, Zbl 0815.35067.

[4] J.M. Arietta, A.N. Carvalho and A. Rodriguez-Bernal, Parabolic problems with nonlinear boundary conditions and critical nonlinearities, J. Differential Equations, 156 (1999), 376-406, MR 2000f:35075, Zbl 0938.35077.

[5] A.N. de Carvalho and J.W. Cholewa, Local well-posedness for strongly damped wave equations with critical nonlinearities, Bull. Austral. Math. Soc., 66 (2002), 443-463.

[6] A.N. de Carvalho, J.W. Cholewa and T. Dlotko, Examples of global attractors in parabolic problems, Hokkaido Math. J., 27 (1998), 77-103, MR 99a:35115, Zbl 0898.35013.

[7] S. Chen and R. Triggiani, Proof of two conjectures of G. Chen and D.L. Russell on structural damping for elastic systems: The case $\alpha=\frac{1}{2}$, Lecture Notes in Mathematics, 1354, Springer, 1988, 234-256, MR 90j:34088, Zbl 0669.34015.

[8] _ Proof of extension of two conjectures on structural damping for elastic systems: The case $\frac{1}{2} \leq \alpha \leq 1$, Pacific J. Math., 136 (1989), 15-55, MR 90g:47071, Zbl 0633.47025.

[9] Characterization of domains of fractional powers of certain operators arising in elastic systems, and applications, J. Differential Equations, 88 (1990), 279-293, MR 91m:47054, Zbl 0717.34066.

[10] J.W. Cholewa and T. Dlotko, Global Attractors in Abstract Parabolic Problems, Cambridge University Press, Cambridge, 2000, MR 2002f:37132, Zbl 0954.35002.

[11] J.K. Hale, Asymptotic Behavior of Dissipative Systems, Amer. Math. Soc., Providence, R.I., 1988, MR 89g:58059, Zbl 0642.58013.

[12] D. Henry, Geometric Theory of Semilinear Parabolic Equations, Springer, Berlin, 1981, MR 83j:35084, Zbl 0456.35001.

[13] P. Massatt, Limiting behavior for strongly damped nonlinear wave equations, J. Differential Equations, 48 (1983), 334-349, MR 85d:47063, Zbl 0561.35049.

[14] H. Triebel, Interpolation Theory, Function Spaces, Differential Operators, Veb Deutscher, Berlin, 1978, MR 80i:4603, Zbl 0387.46033.

[15] G.F. Webb, Existence and asymptotic behavior for a strongly damped nonlinear wave equation, Can. J. Math., 32 (1980), 631-643, MR 81i:35116, Zbl 0432.35046.

[16] S. Zhou, Dimension of the global attractor for strongly damped nonlinear wave equation, J. Math. Anal. Appl., 233 (1999), 102-115, MR 2000e:3515, Zbl 0928.35105. 
Received February 20, 2001. Research of the first author was partially supported by CNPq grant \# 300.889/92-5 and FAPESP grant \# 97/011323-0, Brazil. Research of the second author was partially supported by FAPESP grant \# 99/03116-0, Brazil; also by Polish State Committee for Scientific Research (KBN) grant \# 2 PO3A 03518.

Departamento de Matemática

Instituto de Ciências Matemáticas de SÃo Carlos

Universidade de São Paulo - Campus de São Carlos

Caixa Postal 668

13.560-970 SÃo CARlos SP, Brazil

E-mail address: andcarva@icmc.sc.usp.br

Institute OF MATHEMATiCs

Silesian UNIVERSITY

40-007 Katowice, Poland

E-mail address: jcholewa@ux2.math.us.edu.pl 